\title{
ANALYZING OBSERVATIONS OF MOLECULES IN THE ISM: THEORETICAL AND EXPERIMENTAL STUDIES OF ENERGY TRANSFER
}

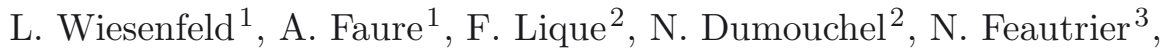 \\ A. Spielfiedel ${ }^{3}$ and Y. Scribano ${ }^{4}$
}

\begin{abstract}
Our laboratories have recently calculated a set of collision coefficients characterizing the efficiency of energy transfer between helium and/or hydrogen and a large variety of interstellar molecules. We have considered with molecules ranging from light hydrides, observed by the Herschel Space Observatory, to medium size molecules, observed by mm antennas, to heavy complex organic molecules, observed also in the $\mathrm{cm}$ range. We present a review of recent theoretical results obtained in our laboratories, for various kinds of commonly observed molecules.
\end{abstract}

\section{Introduction}

Interstellar clouds are mainly composed of hydrogen atoms, hydrogen molecules and helium. Spectra of these species are difficult to record, even if the quadrupole transition of $\mathrm{H}_{2}$ has been observed in many different interstellar media. Major other components are atoms with fine structure transitions and polar molecules, whose rotational and ro-vibrational spectra are readily observed. Among those, $\mathrm{CO}$ transitions are the most widely employed and $\mathrm{H}_{2} \mathrm{O}$ is of particular importance, both because of its prominence in radiative cooling and its obvious role in the chemistry on Earth. However, many other molecules and radicals probe the various states of interstellar gases, from cold pre-stellar cores to highly ionized PDR's and shocked matter or jets.

${ }^{1}$ UJF-Grenoble 1/CNRS, Institut de Planétologie et d'Astrophysique de Grenoble (IPAG) UMR 5274, Grenoble, France; e-mail: laurent.wiesenfeld@obs.ujf-grenoble.fr

${ }^{2}$ LOMC CNRS, Le Havre University, 76058 Le Havre, France

${ }^{3}$ LERMA and UMR 8112 of CNRS, Observatoire de Paris-Meudon, 92195 Meudon Cedex, France

${ }^{4}$ Laboratoire Interdisciplinaire Carnot de Bourgogne-UMR 5209, CNRS-Université de Bourgogne, 21078 Dijon Cedex, France 
The rotational spectra of all these species are routinely observed by $\mathrm{cm}$ to sub-mm telescopes (among those, single dish telescopes like GBT, IRAM, CSO or APEX, or else interferometers like VLA, PdB or the upcoming ALMA). In order to extract quantitative information from the rotational spectra, it has to be determined whether or not the molecule is under Local Thermodynamical Equilibrium (LTE) with the gas or not. If the molecule is in LTE, the kinetic temperature of the gas is equal to the rotational temperature of the molecule. The critical density $n^{*}\left(\mathrm{H}_{2}\right)$ indicates whether the density of colliders $n\left(\mathrm{H}_{2}\right)$ is sufficient to enable LTE. If the molecule is not in LTE, actual collisional coefficients have to be computed, in order to properly quantify the energy transfer between the $\mathrm{H}_{2}$ (or $\mathrm{He}$ ) kinetic energy and the molecule rotational energy.

Our three groups have performed the task of computing collisional coefficients for a large variety of systems. We present a recent list in the next section. A few examples are also discussed in more details, among those water is particularly important.

\section{A list of recent work}

Over the years, in collaboration with several other groups we have performed both PES ab initio computations and dynamical calculations on a large series of systems. A recent list is presented in Table 1 (see also van der Tak 2011). A few highlights are described in the next paragraphs.

Table 1. Some recent computations by our groups.

\begin{tabular}{|c|l||c|l|}
\hline System & Reference & System & Reference \\
& & & \\
\hline & & & \\
$\mathrm{CO}-\mathrm{H}_{2}$ & Wernli (2006) & $\mathrm{NHD}_{2}-\mathrm{H}_{2}$ & Wiesenfeld (2011) \\
$\mathrm{H}_{2} \mathrm{CO}-\mathrm{H}_{2}$ & Troscompt (2009) & $\mathrm{HCOOCH}{ }_{3}-\mathrm{He}$ & Faure (2011) \\
$\mathrm{HC}_{3} \mathrm{~N}-\mathrm{H}_{2}$ & Wernli (2007) & $\mathrm{NH}-\mathrm{He}$ & Tobola (2011) \\
$\mathrm{NH}_{3}-\mathrm{H}_{2}$ & Maret (2009) & $\mathrm{SO}_{2}-\mathrm{H}_{2}$ & Cernicharo (2011) \\
$\mathrm{SiS}-\mathrm{He}$ & Klos (2008) & $\mathrm{CN}-\mathrm{He}$ & Lique (2011) \\
$\mathrm{CN}^{-}-\mathrm{H}_{2}$ & Klos (2011) & $\mathrm{HCN} / \mathrm{HNC}-\mathrm{He}$ & Sarrasin (2010) \\
& & & \\
\hline
\end{tabular}

$\mathbf{S O}_{2}$ [Cernicharo 2011] The quenching of $\mathrm{SO}_{2}$, a molecule abundant in many cold to warm gases, is computed for both ortho- $\mathrm{H}_{2}$ (total nuclear spin $I=1$, odd rotational sates) and para- $\mathrm{H}_{2}(I=0$, even rotational states $)$. While the difference in quenching between ortho- $\mathrm{H}_{2}$ and para- $\mathrm{H}_{2}$ can be very large (more than one order of magnitude) for other polar molecules, $\mathrm{SO}_{2}$ shows a very weak dependence. Also, because of the orientation of the electric dipole with respect to the symmetry axes, metastable states are predicted, yielding maser action in the 
$4_{04}-3_{13}$ transition, as well overcooling (absorption) for the $1_{11}-2_{02}$ transition; overcooling has been actually observed.

HCN-HNC [Sarrasin 2010] The relative abundances of HCN and HNC (hydrogen cyanide and isocyanide) is a long-standing problem. Since no collisional coefficients for the two species were available, Lique et al. (2011) published a comparison of quenching rates in collisions $\mathrm{HCN} / \mathrm{HNC}$ - He. The calculations show very clearly that the collisional propensity rules differ between both species $(\Delta J$ odd is preferred for $\mathrm{HNC}, \Delta J$ even for $\mathrm{HCN}$ ). The effect is very pronounced for HCN: $\Delta J=1,3,5$ is nearly suppressed, even for $T \sim 50 \mathrm{~K}$. The HNC propensity rules are much smoother, and a nearly monotonous decrease is observed for $T=50 \mathrm{~K}, \Delta J=1, \ldots, 5$.

$\mathbf{H C O O C H}_{3}$ [Faure 2011] Methyl formate is the largest abundant complex organic molecule in some warm regions, near proto-stellar corinos in particular. Computing the excitation rates of such a large molecule is a huge challenge. The PES $\mathrm{HCOOCH}_{3}-\mathrm{He}$ was found to be extremely anisotropic, with the methyl-formate molecule being nearly planar. Approaching the He atom in-plane or perpendicular to the plane results is very different interactions, with shorter distances of approach at low temperature ranging from 4.5 au to 3 au. Low temperature rates have been computed. Extending those to higher $T$ remains a difficult task.

Water One of the main motivations for this molecular physics program is the proper computation of excitation and quenching rates of water. Water, in its three forms, $\mathrm{H}_{2} \mathrm{O}, \mathrm{HDO}, \mathrm{D}_{2} \mathrm{O}$ is observed both from the ground (for the deuterated species) and from the space observatory Herschel, HIFI and PACS spectrometers

Table 2. List of recent works of the water quenching. DCS: Differential cross sections; PB: Pressure broadening.

\begin{tabular}{|c|l|l|}
\hline System & Subject & Reference \\
\hline & & \\
$\mathrm{H}_{2} \mathrm{O}-\mathrm{H}_{2}$ & Classical rates & Faure $(2007)$ \\
$\mathrm{H}_{2} \mathrm{O}-\mathrm{H}_{2}$ & Quantum rates & Daniel $(2011)$ \\
$\mathrm{HDO}-\mathrm{H}_{2}$ & Quantum rates & Coutens $(2012)$ \\
& & Faure $(2012)$ \\
$\mathrm{D}_{2} \mathrm{O}-\mathrm{H}_{2}$ & Low- $T$ rates & Vastel $(2010)$, Faure $(2012)$ \\
$\mathrm{H}_{2} \mathrm{O}-\mathrm{H}_{2} / \mathrm{He}$ & DCS & Yang $(2010 \mathrm{a}, \mathrm{b})$ \\
$\mathrm{H}_{2} \mathrm{O}-\mathrm{H}_{2}$ & $\mathrm{~PB}$ & Wiesenfeld $(2010)$ \\
& & Drouin $(2012)$ \\
$\mathrm{D}_{2} \mathrm{O}-\mathrm{D}_{2}$ & Elastic cross sections & Belpassi $(2010)$ \\
$\mathrm{H}_{2} \mathrm{O}-\mathrm{H}_{2}$ & Virial coeff. & Scribano $(2011)$ \\
& & \\
\hline
\end{tabular}


(for $\mathrm{H}_{2} \mathrm{O}$ and $\mathrm{HDO}$ ). A careful quantitative study of water is under way in many sources, by many authors. All recent computations are based on the full PES by Valiron (2008), which allows for inter- and intra-molecular motion. This PES is adapted for all isotopic substitutions. Table 2 gives significant recent computations.

Among those computations, a great number were meant to carefully compare the experimental data with the theoretical predictions. Indeed, since the inferred abundance of water in the ISM depends crucially of the published collision coefficients, the absolute scale is tested in the pressure broadening experiments. Drouin (2012) provides a crucial confirmation of the validity of the PES. Furthermore, since the precision of other PES is comparable to this PES, one gains considerable confidence in the approach presented here. Present-day work consider lacking standard collision partners (like $\mathrm{HCl}, \mathrm{HCO}^{+}$), to open shell radicals as well as to water-water collisions, for cometary comae, is presently under way.

\section{References}

Belpassi, L., et al., 2010, J. Am. Chem. Soc., 132, 13046

Cernicharo, J., et al., 2011, A\&A, 531, A103

Coutens, A., et al., 2012, A\&A, 539, A132

Daniel, F., Dubernet, M.L., \& Grosjean, A., 2011, A\&A, 536, A76

Drouin, B., \& Wiesenfeld, L., 2012, Phys. Rev A., 2012, in print

Faure, A., et al., 2007, A\&A, 472, 1029

Faure, A., Szalewicz, K., \& Wiesenfeld, L., 2011, J. Chem. Phys., 135, 024301

Faure, A., Wiesenfeld, L., Scribano, Y., \& Ceccarelli, C., 2012, MNRAS, 420, 699

Klos, J., \& Lique, F., 2008, MNRAS, 390, 239

Klos, J., \& Lique, F., 2011, MNRAS, 418, 271

Lique, F., \& Klos, J., 2011, MNRAS, 413, L20

Maret, S., Faure, A., Scifoni, E., \& Wiesenfeld, L., 2009, MNRAS, 399, 425

Sarrasin, E., et al., 2010, MNRAS, 404, 518

Scribano, Y., Omololu, A.O., \& Faure, A., 2011, J. Chem. Phys., 135, 116101

Tobola, R., Dumouchel, F., Klos, J., \& Lique, F., 2011, J. Chem. Phys., 134, 024305

Troscompt, N., et al., 2009, A\&A, 493687

Valiron, P., et al., 2008, J. Chem. Phys., 129, 134306

van der Tak, F., 2011, IAU Symp., 280, 449

Vastel, C., et al., 2010, A\&A, 521, L31

Wernli, M., Valiron, P., Faure, A., et al., 2006, A\&A, 446, 367

Wernli, M., et al., 2007, A\&A, 464, 1147 and 475, 391 (erratum)

Wiesenfeld, L., \& Faure, A., 2010, Phys. Rev. A, 82, R040702

Wiesenfeld, L., Scifoni, E., Faure, A., \& Roueff, E., 2011, MNRAS, 413, 509

Yang, C.H., et al., 2010a, J. Phys. Chem. A, 114, 9886

Yang, C.H., et al., 2010b, J. Chem. Phys., 133, 131103 